\title{
SEGURANÇA DO PACIENTE IDOSO RELACIONADA À TERAPÊUTICA MEDICAMENTOSA NO TRATAMENTO DA COVID-19
}

\author{
Francisca Tereza de Galiza' \\ ORCID: 0000-0001-5217-7180 \\ Jessica de Menezes Nogueira' \\ ORCID: 0000-0002-8201-7169 \\ Bruna Karen Cavalcante Fernandes' \\ ORCID: 0000-0003-2808-7526 \\ Angelina Monteiro Furtado' \\ ORCID: 0000-0002-5210-160X
}

'Universidade Federal do Piauí. Teresina, Piauí, Brasil.

\section{Autor Correspondente: \\ Francisca Tereza de Galiza \\ E-mail: terezagaliza@yahoo.com.br}

Como citar:

Galiza FT, Nogueira JM, Fernandes BKC, Furtado AM. Segurança do paciente idoso relacionada à terapêutica medicamentosa no tratamento da covid-19. Segurança do paciente idoso relacionada

à terapêutica medicamentosa no tratamento

da covid-19. In: Santana RF (Org.). Enfermagem gerontologica no cuidado do idoso em tempos da COVID 19. 2.ed.rev. Brasilia, DF: Editora ABEn; 2020.

p 95-100. (Serie Enfermagem e Pandemias, 2). https://doi.org/10.51234/aben.20.e02.c15

\section{INTRODUÇÃO}

A rápida propagação mundial do novo Coronavírus Severe Acute Respiratory Syndrome Coronavirus 2 (SARS-CoV-2), causador da Corona Virus Disease-19 (COVID-19), tem acometido pessoas das diferentes faixas etárias, principalmente entre idosos. A população idosa apresenta maior vulnerabilidade às formas graves da doença e letalidade elevada, em especial idosos frágeis, portadores de comorbidades e residentes de Instituições de Longa Permanência para Idosos (ILPIs).

Os dados da COVID-19 apontam maior taxa de mortalidade entre as pessoas com 80 anos ou mais, em que $14,8 \%$ dos infectados morreram, comparado a $8,0 \%$ entre os idosos de 70 a 79 anos e 8,8\% entre aqueles de 60 a 69 anos (taxa 3,82 vezes maior que a média geral), reforçando as apreensões com a população idosa ${ }^{(1)}$.

Parte relevante dos idosos acometidos pela COVID-19, deverão ser rastreados no primeiro ponto de acesso ao sistema de saúde, logo que apresentarem sintomas da infecção pelo novo coronavírus. Nesse contexto, a atenção primária a saúde possibilita esse tratamento adequado e imediato necessário à população idosa da comunidade, evidenciando uma demanda pelo cuidado gerontológico.

Dentre os aspectos atendidos por meio do cuidado gerontológico, na Atenção Primária à Saúde (APS), destaca-se a polifarmácia, em decorrência do uso excessivo de medicamentos por parte dos idosos, principalmente, os portadores de doenças crônicas. Assim, recomenda-se a revisão das prescrições de medicamentos para reduzir o uso concomitante de cinco ou mais fármacos, bem como prevenir interações medicamentosas e eventos adversos para aqueles que estão sendo tratados com COVID-19(2). Tal cenário mostra-se que para o enfrentamento da COVID-19, considerado problema grave de saúde pública, exigisse medidas efetivas de minimização de riscos para seu enfrentamento, a partir de uma abordagem voltada para a segurança do paciente idoso. 
Entende-se a Segurança do Paciente como sendo a redução, a um mínimo aceitável, do risco de dano desnecessário associado à atenção à saúde. Estima-se que 1 a cada 10 pacientes sofre algum tipo de dano quando recebe prestação de cuidados em um serviço de saúde, e que $50 \%$ destes eventos podem ser evitáveis ${ }^{(3)}$.

Faz-se necessário, portanto, no contexto do novo coronavírus, implementar ações que sensibilizem a equipe multidisciplinar da atenção primária para a cultura da segurança do paciente, visando minimizar agravos quanto a terapêutica medicamentosa junto a pessoa idosa.

\section{OBJETIVO}

Refletir acerca da segurança do paciente idoso relacionada à terapêutica medicamentosa no tratamento da COVID-19.

\section{MÉTODOS}

Trata-se de uma reflexão teórica baseada em estudos científicos e notas técnicas nacionais e internacionais, sobre a segurança do paciente idoso e a terapêutica medicamentosa para COVID-19.

\section{RESULTADOS}

Na sequência, estão apresentadas reflexões pertinentes ao objeto deste estudo, organizadas em categorias, de modo a facilitar a compreensão dos fenômenos que permeiam a segurança do paciente idoso, especialmente relacionada à terapêutica medicamentosa no tratamento da COVID-19.

\section{Vulnerabilidade da pessoa idosa à COVID-19}

Sobre a vulnerabilidade da pessoa idosa no processo de adoecimento da COVID-19, a literatura científica disponível evidencia que alterações fisiológicas do envelhecimento, como a diminuição da reserva funcional do sistema imunológico, e a multimorbidade, que aumentam significativamente o risco para a doença neste grupo etário ${ }^{(4)}$. Alguns estudos apontam, ainda, a idade avançada e a fragilidade como importantes fatores predisponentes à complicações da infecção, com evoluções para a forma grave da doença e morte em maior rapidez se comparada ao jovem ${ }^{(4)}$.

Essas características da senilidade e da senescência humana quando se relacionam à alta virulência do agente etiológico da COVID-19 e ao seu tropismo respiratório requerem do organismo do idoso uma resposta rápida e eficiente para combater o processo infeccioso em curso. No entanto, o que se observa é a inabilidade para a manutenção da homeostase de forma imediata, talvez, associada a imunossenescência, principalmente, na coexistência de outras doenças, expondo, particularmente, a pessoa idosa a agravos e desfechos desfavoráveis relacionados ao novo coronavírus ${ }^{(4)}$.

Um outro fator predisponente a esta vulnerabilidade é a possibilidade da manifestação atípica da infecção. No contexto pandêmico, pesquisas evidenciam que a COVID-19 pode apresentar-se atipicamente com delirium, instabilidade postural ou diarreia e sem febre, potencializando a demora do diagnóstico e agravamento do quadro clínico ${ }^{(5)}$.

Nessa conjuntura, é importante mencionar que a literatura reporta agravos em idosos relacionados à SARS-CoV-2, que incluem a síndrome da doença respiratória aguda, lesão renal aguda, lesão cardíaca e disfunção hepática. Ademais, os pacientes que necessitaram de suporte em unidade de terapia intensiva, por complicação do quadro clínico, apresentaram como comorbidades as doenças cardiovasculares, cerebrovasculares, endócrinas, digestivas e respiratórias ${ }^{(5)}$.

Nesse cenário de fenômenos clínicos para a vulnerabilidade, deve-se acrescentar a inevitável polifarmácia, decorrente da multimorbidade esperada na fase do envelhecimento humano, e da terapia medicamentosa 
para o tratamento da sintomatologia da COVID-19, pois é desconhecido até o momento um antiviral ou vacina específicos para o vírus.

Entretanto, associada a polifarmácia está o risco para um novo fenômeno, a iatrogenia medicamentosa, decursiva de maiores chances de reações adversas e/ou interações medicamentosas, e pelo uso de fármacos que não tenham aprovação oficial para a terapia da SARS-CoV-2(2).

Portanto, o cuidado à saúde da pessoa idosa com COVID-19 deve considerar a interação e compreensão destes fenômenos na tentativa de mitigar o impacto dos mesmos durante o processo de adoecimento pelo novo coronavírus.

\section{Segurança do paciente idoso: terapêutica medicamentosa no tratamento da COVID-19.}

O uso exponencial de medicamentos pelos idosos é decorrente da prevalência de doenças crônicas, dos acometimentos inerentes à idade, da forte influência de venda das indústrias farmacêuticas, além do modelo de medicalização adotado por parte dos profissionais de saúde. Esse consumo amplo tem impacto no âmbito clínico e econômico repercutindo, diretamente, na segurança do paciente.

Observa-se que, os eventos adversos relacionados aos medicamentos se apresentam, com maior frequência, entre o público idoso, tendo como agravante a complexidade da terapia; podendo levar a prejuízos e desfechos negativos. O risco de ocorrência de um evento adverso aumenta em 13\% com o uso de dois agentes, de $58 \%$ quando este número aumenta para cinco, elevando-se para $82 \%$ nos casos em que são consumidos sete ou mais medicamentos(6).

O uso rotineiro ou simultâneo de cinco ou mais medicamentos é um fenômeno conhecido por polifarmácia ou polimedicação, sendo um dos principais problemas relacionados à Segurança do Paciente ${ }^{(6)}$. Acrescenta-se, ainda, que o consumo de múltiplos medicamentos está diretamente associado a um aumento do risco de uso de fármacos potencialmente inapropriados, consequentemente, ao surgimento de eventos adversos, que se potencializam em relação à pessoa idosa. Outros problemas da farmacoterapia, como uso inadequado, interação medicamentosa, iatrogenia, automedicação, dentre outros fenômenos estão relacionados com a introdução crescente e de diferentes terapias farmacológicas. Reconhecer a prática da polimedicação é fator determinante para prevenção do uso irracional de medicamentos ${ }^{(6)}$.

No entanto, com o enfrentamento da COVID-19 e o desenfreado consumo de potenciais terapias, há um risco substancial de ocorrerem interações medicamentosas entre os idosos ${ }^{(5)}$. Muitos desses medicamentos são potencialmente inapropriados e apresentam um risco aumentado de eventos adversos a medicamentos ${ }^{(7)}$.

Com o novo contexto epidemiológico provocado pela COVID-19, parte da população envelhecida, afetada diretamente pelo novo coronavírus, encontra-se sob a supervisão da APS no manejo terapêutico das síndromes gripais, em que as medidas farmacológicas são utilizadas em consonância às recomendações do Ministério da Saúde. Porém, há uma elevada preocupação quanto à automedicação realizada por idosos em seus domicílios sem supervisão da equipe de saúde e de cuidadores.

Dentre as medidas farmacológicas preconizadas está a utilização de antitérmicos por via oral, além do antiviral oseltamivir. Havendo, portanto, a prescrição de fármacos para o controle desses sintomas típicos. Porém, outras medicações estão sendo utilizadas sem as devidas eficácias comprovadas, como a azitromicina, cloroquina e hidroxicloroquina.

Embora com ação anti-inflamatória fraca nas doses terapêuticas e maior ação analgésica e antitérmica, o paracetamol e a dipirona são considerados Anti-Inflamatórios Não Esteroidais (AINEs), classe medicamentosa que pode agravar problemas renais, principalmente em idosos hipertensos e diabéticos, além de aumentar o risco de interações medicamentosas. Estudo brasileiro também identificou a dipirona como o medicamento, dessa classe, mais prescrito para idosos ${ }^{(6)}$.

A Sociedade Americana de Geriatria sugere que o paracetamol deve ser prescrito precedendo os anti-inflamatórios orais. Entretanto, sabe-se que este medicamento possui um metabólito tóxico que pode ser 
acumulado no fígado, vindo a causar, possivelmente, hepatotoxicidade. A dosagem do paracetamol em idosos deve ser individualizada e reduzida, pois seu organismo possui maior dificuldade em eliminar o metabólito ativo do paracetamol, causando maiores danos no fígado, principalmente em pacientes que já possuem comprometimento nesse órgão(7).

Acrescenta-se as interações medicamentosas relacionadas aos AINEs e aos medicamentos de uso contínuo, como agentes hematológicos, antidepressivos, anticonvulsivantes, antihipertensivos e diuréticos frequentemente consumidos pelos idosos com adoecimentos crônicos ${ }^{(6)}$.

Quanto ao uso de oseltamivir, indicado para os casos de síndrome gripal, que tenham situações de risco para complicações, como é o caso das pessoas idosas e/ou portadores de doenças crônicas não transmissíveis $^{(7)}$. Essa recomendação independe da situação vacinal do paciente, mesmo sendo acompanhado pela APS.

Além dos medicamentos descritos acima, muito tem se discutido acerca do uso da cloroquina e hidroxicloroquina na população em geral, mas em especial nos idosos, infectados com COVID-19, devido ao risco de disritmias. Entidades advertem contra o uso desses medicamentos fora de um hospital ou ensaio clínico ${ }^{(8,9)}$.

Quando em uso de cloroquina ou hidroxicloroquina, os médicos devem monitorar o paciente quanto a eventos adversos, especial o intervalo QTc prolongado. A cloroquina em altas doses (600 mg duas vezes ao dia por 10 dias) tem sido associada a toxicidades mais graves. Já a hidroxicloroquina tem menos toxicidades graves (incluindo menor propensão a prolongar o intervalo QTc) e menos interações medicamentosas do que a cloroquina ${ }^{(8,9)}$.

Importante destacar que o efeito colateral mais significativo comum a todos os medicamentos recomendados para COVID-19 é o prolongamento do intervalo QTc ${ }^{(2,5)}$. Em se tratando da combinação desses medicamentos com os psicotrópicos, classe farmacológica comumente usada em idosos, pode ser fatal se não forem tomadas as devidas precauções ${ }^{(7)}$.

Um outro fator a ser conhecido, apesar de se constituir ainda uma hipótese, é o fato de idosos, portadores de comorbidades como Hipertensão Arterial Sistêmica, Diabetes e/ou Doença Renal Crônica, em uso de fármacos Inibidores da Enzima Conversora de Angiotensina (IECA) e Bloqueadores dos Receptores da Angiotensina (BRA), serem mais suscetíveis a infecção pelo novo coronavírus, por estas medicações regularem positivamente o receptor da Enzima Conversora da Angiotensina 2 (ECA2) ${ }^{(6-7)}$.

Portanto, a rotina medicamentosa do idoso, em especial aqueles que estão em isolamento social no domicílio, requer uma avaliação mais cautelosa quanto às interações medicamentosas, no momento da prescrição e dispensação dos fármacos. Nesse contexto, as equipes de saúde devem adotar abordagens centradas no cuidado individualizado ao paciente, a exemplo de estratégias para tomada de decisão clínica, colaboração, adesão ao regime terapêutico e monitoramento(6).

Nesse sentido, para o uso seguro e eficaz dos fármacos, por parte dos idosos, faz-se necessário identificar previamente os problemas relacionados a farmacoterapia e utilizar estratégias de monitoramento de agravos, por meio da abordagem e intervenção terapêutica da equipe multidisciplinar de saúde, que presta assistência ao idoso.

\section{Estratégias de cuidado gerontológico para minimizar agravos provocados por fármacos no contexto pandêmico: boas práticas na atenção primária.}

O mundo acompanha avidamente a evolução científica a respeito de tratamentos efetivos contra o SARS-CoV-2, devido ao seu significativo potencial de infectividade e letalidade, que está gerando números expressivos de mortes no mundo todo.

Assim, é natural que a sociedade anseie por respostas rápidas. Entretanto, apesar de no mundo ter mais de 2.172 estudos clínicos registrados com intuito de avaliar estratégias de tratamento à COVID-19(10), ainda não há evidências científicas robustas que suportem a prescrição farmacológica específica para o novo coronavírus.

A Organização Mundial da Saúde (OMS) e autoridades sanitárias de diversos países, incluindo o Center for Diseases Control (CDC) e o National Institute of Health (NIH) nos Estados Unidos reforçaram que o tratamento medicamentoso para pacientes com COVID-19 seja oferecido somente em cenário de pesquisa clínica ${ }^{(10)}$. 
Contudo, no dia 20 de maio de 2020 o Ministério da Saúde publicou orientações relacionadas ao uso permissivo de medicamentos, mesmo em pacientes com doença leve ou moderada, orientando o uso precoce de cloroquina ou hidroxicloroquina em associação com azitromicina ${ }^{(8)}$.

Essas orientações devem ser vistas com extrema cautela pelos profissionais da equipe de saúde, em especial a da APS, no momento da Avaliação Geriátrica Ampla, relacionando as particularidades inerentes ao processo de envelhecer à prescrição desses medicamentos em idosos com COVID-19 na fase leve e/ou moderada, sobretudo naqueles que são frágeis e apresentam comorbidades ${ }^{(5)}$.

Diante disso, salienta-se que o cuidado ao idoso exige um olhar diferenciado por parte dos profissionais de saúde, a fim de adotar a farmacoterapia mais apropriada e segura possível, respeitando as indicações, doses, interações e contraindicações de cada medicamento, considerando critérios pré-definidos e melhores evidências disponíveis ${ }^{(7)}$.

Nesse contexto, boas práticas de cuidados aos idosos são necessárias e devem ser adotadas pelos profissionais de saúde da APS, tais como: Estabelecer um plano terapêutico seguro; Elaborar esquemas terapêuticos simples; Utilizar uma escrita legível nas prescrições, se possível ser digitada; Monitorar possíveis duplicações e as interações medicamentosas; Planejar a renovação de receituários, pelo maior período possível, para idosos com doenças crônicas estáveis; Ponderar os riscos e benefícios dos medicamentos para o idoso; e Esclarecer os riscos de medicamentos de alta vigilância.

Acrescenta-se ainda, outras práticas de cuidado, como: Reforçar aos cuidadores a importância do estabelecimento de uma parceria; Melhorar a comunicação e tornar as relações terapêuticas mais horizontais; Educar o idoso e cuidador sobre os riscos da automedicação; Considerar as peculiaridades do envelhecimento ao recomendar um tratamento; Pesquisar e adotar na assistência o uso de dispositivos/tecnologias que facilitem a identificação das drogas e auxiliem os idosos a lembrar dos horários e das doses; Incorporar na prática clínica o conhecimento sobre multimorbidade e polifarmácia; e Manter os conhecimentos atualizados sobre a terapêutica medicamentosa para o idoso, especialmente relacionada à COVID-19.

Para melhorar a segurança no uso de medicamentos, recomenda-se, ainda, uma revisão das prescrições de medicamentos, considerando os dados em prontuário e as informações fornecidas pelos idosos durante os atendimentos, para reduzir a polifarmácia, prevenir interações medicamentosas e eventos adversos aos que estão sendo tratados com COVID-19(2). Outra estratégia é investigar os medicamentos adquiridos pelo próprio idoso, sem prescrição, caracterizando, assim, a automedicação.

Com vistas a minimizar esse fenômeno da automedicação, bem como da polifarmácia, orienta-se solicitar ao idoso que, na próxima consulta, leve consigo todos os medicamentos que costuma utilizar frequente e ocasionalmente. Com essa medida simples é possível detectar o uso de automedicação, a utilização de posologia incorreta e de mais de um medicamento para o mesmo objetivo, às vezes prescrito por profissionais diferentes.

\section{Limitações}

A restrita literatura sobre posologia, indicações e efeitos adversos das medicações para tratamento da COVID-19 e as considerações gerontológicas limitam a discussão ao mesmo tempo que ressalta a necessidade de estudos que abordem a temática

\section{Implicações para a prática de enfermagem}

A utilização criteriosa e cautelosa dos medicamentos, quanto à dose, tipo e intervalos, bem como adesão aos medicamentos, suscita a necessidade de orientação das pessoas idosas e seus familiares, como elementos essenciais na manutenção da qualidade de vida do idoso ${ }^{(7)}$. Racionalizar o uso de medicamentos e evitar os agravos advindos da polifarmácia, iatrogenia e automedicação em idosos são um dos grandes desafios da saúde pública em tempos de pandemia pela COVID-19. 


\section{CONSIDERAÇÕES FINAIS}

A equipe multidisciplinar, responsável pelo cuidado à saúde da pessoa idosa com COVID-19, deve ter ainda mais atenção aos fatores inerentes à senescência e senilidade, para que se tente diminuir os impactos dos mesmos no processo de adoecimento desencadeado pelo SARS-CoV-2.

Nesse sentido, a utilização racional de fármacos pelos idosos deve ser vista de forma especial pela equipe de saúde, com o fim de prever problemas advindos da polifarmácia, iatrogenia e automedicação, além de utilizar estratégias que monitorem os agravos que possam ter como cerne a farmacoterapia.

\section{AGRADECIMENTO}

\section{Ao Departamento Científico de Enfermagem Gerontológica da ABEn Nacional.}

\section{REFERÊNCIAS}

1. Hammerschmidt KSA, Santana RF. Saúde do idoso em tempos de pandemia Covid-19. Cogitare enferm. 2020; 25. doi:10.5380/ce.v25i0.72849.

2. World Health Organization (WHO). Clinical management of COVID-19. [Internet] 2020 [citado em 13 de junho de 2020]. Disponível em: https://www.who.int/publications/i/item/clinical-management-of-covid-19

3. Runciman WHP, Thomson R, Schaaf TV, Sherman H, Lewalle P. Towards an International Classification for Patient Safety: key concepts and terms. Int J Qual Health Care. 2009; 21(1):18-26. doi: 10.1093/intqhc/mzn057

4. Hubbard RE, Maier AB, Hilmer SN, Naganathan V, Etherton-Beer C, Rockwood K. Frailty in the face of COVID-19. Age and Ageing. 2020; afaa095. doi: 10.1093/ageing/afaa095.

5. Koeberle S, Tannou T, Bouiller K, Becoulet N, Outrey J, Chirouze C, Aubry R. COVID 19 outbreak: organisation of a geriatric assessment and coordination unit: a French example. Age and Agein. 2020; afaa092. doi: 10.1093/ageing/afaa092.

6. Araújo LU, Santos DF, Bodevan EC, Cruz HL, Souza J, Silva-Barcellos NM. Segurança do paciente e polimedicação na Atenção Primária à Saúde: pesquisa transversal em pacientes com doenças crônicas. Rev. Latino-Am. Enfermagem. 2019; 27: e3217. doi: 10.1590/1518-8345.3123.3217.

7. American Geriatrics Society Beers Criteria Update Expert P. American Geriatrics Society 2019 Updated AGS Beers Criteria(R) for Potentially Inappropriate Medication Use in Older Adults. J Am Geriatr Soc. 2019;67(4):674-694. doi: 10.1111/jgs.15767.

8. Brasil. Ministério da Saúde. Orientações do ministério da saúde para manuseio medicamentoso precoce de pacientes com diagnóstico da covid-19. [Internet] 2020 [Citado em 12 de junho de 2020]. Disponível em: https://www.saude.gov.br/ images/pdf/2020/May/20/orientacoes-manuseio-medicamentoso-covid19.pdf

9. Pastick KA, Okafor EC, Wang F, et al. Review: Hydroxychloroquine and Chloroquine for Treatment of SARS-CoV-2 (COVID-19). Open Forum Infectious Diseases. 2020. 7(4); ofaa130. doi: 10.1093/ofid/ofaa130

10. National Institute of Health (NIH). COVID-19 Treatment Guidelines - Potential Antiviral Drugs Under Evaluation for the Treatment of COVID-19. [Internet] 2020 [Citado em 13 de junho de 2020]. Disponível em: https://www. covid19treatmentguidelines.nih.gov/antiviral-therapy/ 